International Journal of Applied Mathematics

Volume 34 No. $4 \quad 2021,693-704$

ISSN: 1311-1728 (printed version); ISSN: 1314-8060 (on-line version)

doi: http://dx.doi.org/10.12732/ijam.v34i4.8

\title{
STABILITY OF BINET-CAUCHY FUNCTIONAL EQUATION ON NON-ARCHIMEDEAN BANACH ALGEBRAS
}

Karthikeyan Subramani ${ }^{1}$, P. Palani ${ }^{2}$

${ }^{1}$ Department of Mathematics

R.M.K. Engineering College

Kavaraipettai - 601206, Tamil Nadu, INDIA

${ }^{2}$ Department of Mathematics

Sri Vidya Mandir Arts and Science College

Uthangarai-636902, Tamil Nadu, INDIA

Abstract: In this paper, we introduced Binet-Cauchy additive functional equation

$$
\begin{aligned}
\left(\sum_{i=1}^{n} f\left(x_{i} y_{i}\right)\right) & \left(\sum_{j=1}^{n} f\left(u_{j} v_{j}\right)\right)=f\left(\sum_{i=1}^{n} x_{i} v_{i}\right) f\left(\sum_{j=1}^{n} y_{j} u_{j}\right) \\
& +\sum_{1 \leq i<j \leq n} f\left(x_{i} u_{j}-x_{j} u_{i}\right) f\left(y_{i} v_{j}-y_{j} v_{i}\right)
\end{aligned}
$$

and established related generalized Ulam-Hyers stability results in non-Archimedean Banach modules over a Banach algebra.

AMS Subject Classification: 39B52, 32B72, 32B82

Key Words: functional equation; generalized Ulam-Hyers stability; Banach algebra

\section{Introduction}

The study of stability problems for functional equations is related to a question of Ulam [27] concerning the stability of group homomorphisms and affirma-

Received: February 9, 2021

(c) 2021 Academic Publications

${ }^{\S}$ Correspondence author 
tively answered for Banach spaces by Hyers [14]. It was further generalized and excellent results obtained by number of authors as $[2,13,23,24,26]$.

The functional equation

$$
f(x+y)=f(x)+f(y)
$$

is said to be additive functional equation because the additive function $f(x)=a x$ is a solution of the functional equation (1).

Let $\mathbf{K}$ denote a field and function (valuation absolute) $|$.$| from \mathrm{K}$ into $[0, \infty)$. A non-Archimedean valuation is a function $|$.$| that satisfies the strong$ triangle inequality; namely, $|x+y| \leq \max \{|x|,|y|\} \leq|x|+|y|$ for all $x, y \in \mathbf{K}$. The associated field $\mathbf{K}$ is referred to as a non-Archimedean field. Clearly, $|1|=$ $|-1|=1$ and $|n| \leq 1$ for all $n \geq 1$. A trivial example of a non-Archimedean valuation is the function $|$.$| taking everything except 0$ into 1 and $|0|=0$. We always assume in addition that $|$.$| is nontrivial, i.e., there is a z \in \mathbf{K}$ such that $|z| \neq 0,1$.

Let $X$ be a linear space over a field $\mathbf{K}$ with a non-Archimedean nontrivial valuation $|$.$| . A function \|\|:. X \rightarrow[0, \infty)$ is said to be a non-Archimedean norm if it is a norm over $\mathbf{K}$ with the strong triangle inequality (ultrametric); namely, $\|x+y\| \leq \max \{\|x\|,\|y\|\}$ for all $x, y \in X$. Then $(X,\|\cdot\|)$ is called a non-Archimedean space.

In any such a space a sequence $\left\{x_{n}\right\}_{n \in \mathbf{N}}$ is Cauchy if and only if $\left\{x_{n+1}-\right.$ $\left.x_{n}\right\}_{n \in \mathbf{N}}$ converges to zero. By a complete non-Archimedean space we mean one in which every Cauchy sequence is convergent. A non-Archimedean Banach algebra is a complete non-Archimedean algebra $\mathcal{A}$ which satisfies $\|x y\| \leq\|x\|\|y\|$ for all $x, y \in \mathcal{A}$.

In this paper, we investigated the general solution in vector spaces and discussed the generalized Ulam-Hyers stability of Binet-Cauchy functional equation

$$
\begin{aligned}
\left(\sum_{i=1}^{n} f\left(x_{i} y_{i}\right)\right) & \left(\sum_{j=1}^{n} f\left(u_{j} v_{j}\right)\right)=f\left(\sum_{i=1}^{n} x_{i} v_{i}\right) f\left(\sum_{j=1}^{n} y_{j} u_{j}\right)+ \\
& \sum_{1 \leq i<j \leq n} f\left(x_{i} u_{j}-x_{j} u_{i}\right) f\left(y_{i} v_{j}-y_{j} v_{i}\right)
\end{aligned}
$$

in non-Archimedean Banach modules over a Banach algebra. 


\section{General Solution of the Functional Equation (2)}

In this section, we investigate the general solution of the functional equation (2). Throughout this section let us consider $X$ and $Y$ be real vector spaces.

Theorem 1. Let $X$ and $Y$ be real vector spaces. If the mapping $f: X \rightarrow$ $Y$ satisfies the functional equation (2) for all $x_{1}, y_{1}, u_{1}, v_{1}, \cdots, x_{n}, y_{n}, u_{n}, v_{n} \in X$ then $f: X \rightarrow Y$ satisfying the functional equation (1) for all $x, y \in X$.

Proof. Setting $\left(x_{3}, y_{3}, u_{3}, v_{3}, \cdots, x_{n}, y_{n}, u_{n}, v_{n}\right)$ by $(0,0, \cdots, 0,0)$ in 2 , we get

$$
\begin{aligned}
& \left(f\left(x_{1} y_{1}\right)+f\left(x_{2} y_{2}\right)\right)\left(f\left(u_{1} v_{1}\right)+f\left(u_{2} v_{2}\right)\right) \\
& =f\left(x_{1} v_{1}+x_{2} v_{2}\right) f\left(y_{1} u_{1}+y_{2} v_{2}\right)+f\left(x_{1} u_{2}-x_{2} u_{1}\right) f\left(y_{1} v_{2}-y_{2} v_{1}\right)
\end{aligned}
$$

for all $x_{1}, y_{1}, u_{1}, v_{1}, x_{2}, y_{2}, u_{2}, v_{2} \in X$.

Replacing $\left(x_{1}, y_{1}, u_{1}, v_{1}, x_{2}, y_{2}, u_{2}, v_{2}\right)$ by $\left(x^{\frac{1}{4}}, x^{\frac{1}{4}}, \cdots, x^{\frac{1}{4}}, x^{\frac{1}{4}}\right)$ in $(3)$, we obtain

$$
n^{2}(f(\sqrt{x}))^{2}=(f(n \sqrt{x}))^{2}
$$

for all $x \in X$. Setting $u_{1}=0$ and $v_{1}=0$ in (3), we get

$$
(f(x))^{2}=f\left(x^{2}\right)
$$

for all $x \in X$. Replacing $x$ by $\sqrt{x}$ in (5), we arrive

$$
(f(\sqrt{x}))^{2}=f(x)
$$

for all $x \in X$. Using (6) in (4), we reach

$$
n^{2}(f(x))=\left(f\left(n^{2} x\right)\right)
$$

for all $x \in X$. Put $n^{2}=k$ in above equation (7), we get

$$
k(f(x))=(f(k x))
$$

for all $x \in X$. Taking $x_{1}=v_{1}=0$ and $x_{2}=y_{1}=y_{2}=u_{1}=u_{2}=v_{2}=\sqrt{x}$ in (4) and using (8), we obtain

$$
f(-x)=-f(x)
$$

for all $x \in X$. Therefore $f$ is an odd function. Letting $x_{1}=y_{1}=u_{2}=v_{2}=\sqrt{x}$; $x_{2}=y_{2}=-\sqrt{y} ; u_{1}=\sqrt{y}$ and $v_{1}=0$ in (4), we derive (1). Hence completes the proof. 


\section{Stability Results}

In this section, we present the generalized Ulam - Hyers stability of the functional equation (2).

Throughout this section, let $\mathcal{B}$ be a Banach-algebra with norm $\|\cdot\|_{A}$ and ${ }_{\mathcal{B}} X$ be left Banach $\mathcal{B}$-module with norm $\|$.$\| and and { }_{\mathcal{B}} Y$ be non-Archimedean left Banach $\mathcal{B}$-module with norm $\|$. $\|$. Define a mapping $F:_{\mathcal{B}} X \rightarrow_{\mathcal{B}} Y$ by

$$
\begin{gathered}
F\left(x_{1}, y_{1}, u_{1}, v_{1}, \cdots, x_{n}, y_{n}, u_{n}, v_{n}\right)=\left(\sum_{i=1}^{n} f\left(x_{i} y_{i}\right)\right)\left(\sum_{j=1}^{n} f\left(u_{j} v_{j}\right)\right) \\
-f\left(\sum_{i=1}^{n} x_{i} v_{i}\right) f\left(\sum_{j=1}^{n} y_{j} u_{j}\right) \\
-\sum_{1 \leq i<j \leq n} f\left(x_{i} u_{j}-x_{j} u_{i}\right) f\left(y_{i} v_{j}-y_{j} v_{i}\right)
\end{gathered}
$$

for all $x_{1}, y_{1}, u_{1}, v_{1}, \cdots, x_{n}, y_{n}, u_{n}, v_{n} \in_{\mathcal{B}} X$.

Theorem 2. Let $j \in\{-1,1\}$. Let $\alpha: \mathcal{B} X^{4 n} \rightarrow[0, \infty)$ be a function such that

$$
\begin{aligned}
& \sum_{k=0}^{\infty} \frac{\alpha\left(n^{k j} x_{1}, n^{k j} y_{1}, \cdots, n^{k j} u_{n}, n^{k j} v_{n}\right)}{n^{k j}}, \text { converges to } \Re \text { and } \\
& \lim _{k \rightarrow \infty} \frac{\alpha\left(n^{k j} x_{1}, n^{k j} y_{1}, \cdots, n^{k j} u_{n}, n^{k j} v_{n}\right)}{n^{k j}}<\infty
\end{aligned}
$$

for all $x_{1}, y_{1}, u_{1}, v_{1}, \cdots, x_{n}, y_{n}, u_{n}, v_{n} \in_{\mathcal{B}} X$ and let $f: \mathcal{B} X \rightarrow_{\mathcal{B}} Y$ be a function satisfies the inequality

$$
\left\|F\left(x_{1}, y_{1}, \cdots, u_{n}, v_{n}\right)\right\| \leq \alpha\left(x_{1}, y_{1}, \cdots, u_{n}, v_{n}\right)
$$

for all $x_{1}, y_{1}, u_{1}, v_{1}, \cdots, x_{n}, y_{n}, u_{n}, v_{n} \in_{\mathcal{B}} X$ and

$$
\begin{array}{r}
\left\|f\left(x_{1} y_{1} \cdots u_{n} v_{n}\right)-f\left(x_{1}\right) f\left(y_{1}\right) \cdots f\left(u_{n}\right) f\left(v_{n}\right)\right\| \\
\leq \alpha\left(x_{1}, y_{1}, u_{1}, v_{1}, \cdots, x_{n}, y_{n}, u_{n}, v_{n}\right)
\end{array}
$$

for all $x, y \in \mathcal{B} X$. Then there exists a unique additive homomorphism $\mathcal{H}: \mathcal{B}$ $X \rightarrow \mathcal{B} Y$ such that

$$
\|f(x)-\mathcal{H}(x)\|
$$




$$
\leq \frac{1}{|k|} \max \left\{\frac{1}{|k|^{i}} \alpha\left(\left(k^{i} x\right)^{\frac{1}{4}}, \cdots,\left(k^{i} x\right)^{\frac{1}{4}}\right): 0 \leq j<n\right\}
$$

for all $x \in_{\mathcal{B}} X$. The mapping $\mathcal{H}(x)$ is defined by

$$
\mathcal{H}(x)=\lim _{m \rightarrow \infty} \frac{f\left(k^{m j} x\right)}{|k|^{m j}}
$$

for all $x \in \mathcal{B} X$.

Proof. Assume $j=1$. Replacing $\left(x_{1}, y_{1}, \cdots, x_{n}, y_{n}, u_{n}, v_{n}\right)$ by $\left(x^{\frac{1}{4}}, x^{\frac{1}{4}}\right.$, $\left.x^{\frac{1}{4}}, x^{\frac{1}{4}}, \cdots, x^{\frac{1}{4}}, x^{\frac{1}{4}}\right)$ and using $(5)$ in (11), we get

$$
\left\|n^{2} f(x)-f\left(n^{2} x\right)\right\| \leq \alpha\left(x^{\frac{1}{4}}, \cdots, x^{\frac{1}{4}}\right)
$$

for all $x \in_{\mathcal{B}} X$. Above equation (15) can be rewritten as

$$
\left\|f(x)-\frac{f(k x)}{k}\right\| \leq \frac{1}{|k|} \alpha\left(x^{\frac{1}{4}}, \cdots, x^{\frac{1}{4}}\right),
$$

where $k=n^{2}$, for all $x \in_{\mathcal{B}} X$. Replacing $x$ by $k x$ in (16) and divided by $k$, we get

$$
\left\|\frac{f(k x)}{k}-\frac{f\left(k^{2} x\right)}{k^{2}}\right\| \leq \frac{1}{|k|^{2}} \alpha\left((k x)^{\frac{1}{4}}, \cdots,(k x)^{\frac{1}{4}}\right)
$$

for all $x \in_{\mathcal{B}} X$. Combining (16) and (17), we obtain

$$
\begin{aligned}
& \left\|f(x)-\frac{f\left(k^{m} x\right)}{k^{m}}\right\| \\
& \quad \leq \frac{1}{|k|} \max \left\{\alpha\left(x^{\frac{1}{4}}, \cdots, x^{\frac{1}{4}}\right), \alpha\left((k x)^{\frac{1}{4}}, \cdots,(k x)^{\frac{1}{4}}\right)\right\}
\end{aligned}
$$

for all $x \in_{\mathcal{B}} X$. Using induction on a positive integer $m$, we obtain that

$$
\begin{aligned}
& \left\|f(x)-\frac{f\left(k^{m} x\right)}{k^{m}}\right\| \\
& \leq \frac{1}{|k|} \max \left\{\frac{1}{|k|^{i}} \alpha\left(\left(k^{i} x\right)^{\frac{1}{4}}, \cdots,\left(k^{i} x\right)^{\frac{1}{4}}\right): 0 \leq i<m\right\}
\end{aligned}
$$

for all $x \in_{\mathcal{B}} X$. In order to prove the convergence of the sequence $\left\{\frac{f\left(k^{m} x\right)}{k^{m}}\right\}$, replace $x$ by $k^{l} x$ and divided by $k^{l}$ in (19), for any $l, m>0$, we arrive to

$$
\left\|\frac{f\left(k^{l} x\right)}{k^{l}}-\frac{f\left(k^{l+m} x\right)}{k^{l+m}}\right\|
$$




$$
\leq \frac{1}{|k|} \max \left\{\frac{1}{|k|^{i+l}} \alpha\left(\left(k^{i+l} x\right)^{\frac{1}{4}}, \cdots,\left(k^{i+l} x\right)^{\frac{1}{4}}\right): 0 \leq i<m\right\}
$$

for all $x \in \in_{\mathcal{B}} X$. Since the right hand side of the inequality (20) tends to 0 as $m \rightarrow \infty$, the sequence $\left\{\frac{f\left(k^{m} x\right)}{k^{m}}\right\}$ is a Cauchy sequence. Since ${ }_{\mathcal{B}} Y$ is complete, there exists a mapping $\mathcal{H}: \mathcal{B} X \rightarrow_{\mathcal{B}} Y$ such that

$$
\mathcal{H}(x)=\lim _{m \rightarrow \infty} \frac{f\left(k^{m} x\right)}{k^{m}}, \forall x \in_{\mathcal{B}} X .
$$

Letting $m \rightarrow \infty$ in (19), we see that (13) holds for all $x \in_{\mathcal{B}} X$. Now we need to prove $\mathcal{H}$ satisfies $(2)$, replacing $\left(x_{1}, y_{1}, \cdots, u_{n}, v_{n}\right)$ by $\left(k^{m} x_{1}, k^{m} y_{1}, \cdots, k^{m} u_{n}\right.$, $k^{m} v_{n}$ ) and divided by $k^{m}$ in (11), we arrive

$$
\begin{aligned}
& \frac{1}{|k|^{m}}\left\|F\left(k^{m} x_{1}, k^{m} y_{1}, k^{m} u_{1}, k^{m} v_{1}, \cdots, k^{m} x_{n}, k^{m} y_{n}, k^{m} u_{n}, k^{m} v_{n}\right)\right\| \\
& \leq \frac{1}{|k|^{m}} \alpha\left(k^{m} x_{1}, k^{m} y_{1}, k^{m} u_{1}, k^{m} v_{1}, \cdots, k^{m} x_{n}, k^{m} y_{n}, k^{m} u_{n}, k^{m} v_{n}\right)
\end{aligned}
$$

for all $x_{1}, y_{1}, u_{1}, v_{1}, \cdots, x_{n}, y_{n}, u_{n}, v_{n} \in_{\mathcal{B}} X$. Letting $m \rightarrow \infty$ in the above inequality, we arrive

$$
\left\|\mathcal{H}\left(k^{m} x_{1}, k^{m} y_{1}, k^{m} u_{1}, k^{m} v_{1}, \cdots, k^{m} x_{n}, k^{m} y_{n}, k^{m} u_{n}, k^{m} v_{n}\right)\right\|=0 .
$$

Hence $\mathcal{H}$ satisfies (2) for all $x_{1}, y_{1}, u_{1}, v_{1}, \cdots, x_{n}, y_{n}, u_{n}, v_{n} \in_{\mathcal{B}} X$. This shows that $\mathcal{H}$ is additive. Also

$$
\begin{aligned}
& \|\mathcal{H}(x y)-\mathcal{H}(x) \mathcal{H}(y)\| \\
& =\lim _{m \rightarrow \infty} \frac{1}{|k|^{2 m}}\left\|f\left(k^{m} x k^{m} y\right)-f\left(k^{m} x\right) f\left(k^{m} y\right)\right\| \\
& \leq \lim _{m \rightarrow \infty} \frac{1}{|k|^{2 m}} \alpha\left(\left(k^{m} x\right)^{\frac{1}{2}}, \cdots,\left(k^{m} y\right)^{\frac{1}{2}}, 0, \cdots, 0\right)=0
\end{aligned}
$$

for all $x, y \in_{\mathcal{B}} X$. Therefore, $\mathcal{H}$ is additive homomorphism. In order to prove $\mathcal{H}$ is unique, let $\mathcal{H}^{\prime}(x)$ be another additive mapping satisfying (13) and (2). Then

$$
\begin{aligned}
& \left\|\mathcal{H}(x)-\mathcal{H}^{\prime}(x)\right\|=\frac{1}{|k|^{m}}\left\|\mathcal{H}\left(k^{m} x\right)-\mathcal{H}^{\prime}\left(k^{m} x\right)\right\| \\
& \leq \frac{1}{|k|^{m}} \max \left\{\left\|\mathcal{H}\left(k^{m} x\right)-f\left(k^{m} x\right)\right\|,\left\|f\left(k^{m} x\right)-\mathcal{H}^{\prime}\left(k^{m} x\right)\right\|\right\} \\
& \leq \frac{1}{|k|} \max \left\{\frac{1}{|k|^{i}} \alpha\left(\left(k^{i} x\right)^{\frac{1}{4}}, \cdots,\left(k^{i} x\right)^{\frac{1}{4}}\right): 0 \leq i<m\right\}
\end{aligned}
$$




$$
\rightarrow 0 \text { as } m \rightarrow \infty
$$

for all $x \in_{\mathcal{B}} X$. Hence $\mathcal{H}$ is unique.

For $j=-1$, we can prove the similar stability result. This completes the proof of the theorem.

The following corollary is an immediate consequence of Theorem 2 concerning the stability of (2).

Corollary 3. Let $\lambda$ and $s$ be nonnegative real numbers. If a function $f: \mathcal{B} X \rightarrow_{\mathcal{B}} Y$ satisfies the inequality

$$
\begin{aligned}
& \left\|F\left(x_{1}, y_{1}, u_{1}, v_{1} \cdots, x_{n}, y_{n}, u_{n}, v_{n}\right)\right\| \\
& \leq\left\{\begin{array}{l}
\lambda, \\
\lambda \sum_{i=1}^{n}\left\{\left\|x_{i}\right\|^{s}+\left\|y_{i}\right\|^{s}+\left\|u_{i}\right\|^{s}+\left\|v_{i}\right\|^{s}\right\}, s \neq 1 ; \\
\lambda\left\{\prod_{i=1}^{n}\left\|x_{i}\right\|^{s}\left\|y_{i}\right\|^{s}\left\|u_{i}\right\|^{s}\left\|v_{i}\right\|^{s}\right\}, s \neq \frac{1}{4 n} ; \\
\lambda\left\{\prod_{i=1}^{n}\left\|x_{i}\right\|^{s}\left\|y_{i}\right\|^{s}\left\|u_{i}\right\|^{s}\left\|v_{i}\right\|^{s}\right. \\
\left.+\sum_{i=1}^{n}\left(\left\|x_{i}\right\|^{4 n s}+\left\|y_{i}\right\|^{4 n s}+\left\|u_{i}\right\|^{4 n s}+\left\|v_{i}\right\|^{4 n s}\right)\right\}, s \neq \frac{1}{4 n}
\end{array}\right.
\end{aligned}
$$

for all $x_{1}, y_{1}, u_{1}, v_{1} \cdots, x_{n}, y_{n}, u_{n}, v_{n} \in_{\mathcal{B}} X$ and

$$
\begin{aligned}
& \left\|f\left(x_{1} y_{1} \cdots u_{n} v_{n}\right)-f\left(x_{1}\right) f\left(y_{1}\right) \cdots f\left(u_{n}\right) f\left(v_{n}\right)\right\| \\
& \leq\left\{\begin{array}{l}
\lambda, \\
\lambda\left\{\left\|x_{i}\right\|^{s}+\left\|y_{i}\right\|^{s}+\left\|u_{i}\right\|^{s}+\left\|v_{i}\right\|^{s}\right\}, \\
\lambda\left\{\prod_{i=1}^{n}\left\|x_{i}\right\|^{s}\left\|y_{i}\right\|^{s}\left\|u_{i}\right\|^{s}\left\|v_{i}\right\|^{s}\right\}, \\
\lambda\left\{\prod_{i=1}^{n}\left\|x_{i}\right\|^{s}\left\|y_{i}\right\|^{s}\left\|u_{i}\right\|^{s}\left\|v_{i}\right\|^{s}\right. \\
\left.+\left(\left\|x_{i}\right\|^{4 n s}+\left\|y_{i}\right\|^{4 n s}+\left\|u_{i}\right\|^{4 n s}+\left\|v_{i}\right\|^{4 n s}\right)\right\},
\end{array}\right.
\end{aligned}
$$

for all $x_{1}, y_{1}, u_{1}, v_{1} \cdots, x_{n}, y_{n}, u_{n}, v_{n} \in_{\mathcal{B}} X$. Then there exists a unique additive 
homomorphism $\mathcal{H}: \mathcal{B} X \rightarrow_{\mathcal{B}} Y$ such that

$$
\|f(x)-\mathcal{H}(x)\| \leq\left\{\begin{array}{l}
\frac{\lambda}{|k-1|}, \\
\frac{\left.4 n \lambda|| x\right|^{s}}{\left|k-k^{s}\right|}, \\
\frac{\lambda|| x||^{4 n s}}{\left|k-k^{4 n s}\right|} \\
\frac{5 n \lambda|| x||^{4 n s}}{\left|k-k^{4 n s}\right|}
\end{array}\right.
$$

for all $x \in \in_{\mathcal{B}} X$, where $k=n^{2}$.

Theorem 4. Let $j \in\{-1,1\}$. Let $\alpha:_{\mathcal{B}} X^{4 n} \rightarrow[0, \infty)$ be a function such that (10) for all $x_{1}, y_{1}, u_{1}, v_{1}, \cdots, x_{n}, y_{n}, u_{n}, v_{n} \in_{\mathcal{B}} X$ and let $f: \mathcal{B} X \rightarrow_{\mathcal{B}} Y$ be a function satisfies the inequality (11) for all $x_{1}, y_{1}, u_{1}, v_{1}, \cdots, x_{n}, y_{n}, u_{n}, v_{n} \in_{\mathcal{B}} X$ and

$$
\begin{aligned}
& \| f\left(x_{1} y_{1} \cdots u_{n} v_{n}\right)-f\left(x_{1}\right) y_{1} \cdots u_{n} v_{n}-x_{1} f\left(y_{1}\right) \cdots \\
& u_{n} v_{n}-\cdots-x_{1} y_{1} \cdots u_{n} f\left(v_{n}\right) \| \leq \alpha\left(x_{1}, y_{1}, \cdots, u_{n}, v_{n}\right)
\end{aligned}
$$

for all $x, y \in_{\mathcal{B}} X$. Then there exists a unique additive derivation $\mathcal{D}:_{\mathcal{B}} X \rightarrow_{\mathcal{B}} X$ such that

$$
\begin{aligned}
& \|f(x)-\mathcal{D}(x)\| \\
& \leq \frac{1}{|k|} \max \left\{\frac{1}{|k|^{i}} \alpha\left(\left(k^{i} x\right)^{\frac{1}{4}}, \cdots,\left(k^{i} x\right)^{\frac{1}{4}}\right): 0 \leq j<n\right\}
\end{aligned}
$$

for all $x \in_{\mathcal{B}} X$. The mapping $\mathcal{D}(x)$ is defined by

$$
\mathcal{D}(x)=\lim _{m \rightarrow \infty} \frac{f\left(k^{m j} x\right)}{|k|^{m j}}
$$

for all $x \in \mathcal{B} X$.

Proof. Assume $j=1$. By the same reasoning as that in the proof of the Theorem 2, there exist a unique additive mapping $\mathcal{D}: \mathcal{B} X \rightarrow_{\mathcal{B}} X$ satisfying (25). The mapping $\mathcal{D}: \mathcal{B} X \rightarrow_{\mathcal{B}} X$ given by $\mathcal{D}(x)=\lim _{k \rightarrow \infty} \frac{f\left(n^{k} x\right)}{n^{k}}$. It follows from (11) that

$$
\|\mathcal{D}(x y)-[x \mathcal{D}(y)]-[\mathcal{D}(x) y]\|
$$




$$
\begin{aligned}
& =\lim _{k \rightarrow \infty} \frac{1}{n^{2 k}}\left\|f\left(n^{2 k} x y\right)-\left(n^{k} x\right) f\left(n^{k} y\right)-f\left(n^{k} x\right)\left(n^{k} y\right)\right\| \\
& \leq \lim _{k \rightarrow \infty} \frac{1}{n^{2 k}} \alpha\left(n^{k} x, n^{k} y, 0, \cdots, 0\right)=0
\end{aligned}
$$

for all $x, y \in_{\mathcal{B}} X$. Therefore $\mathcal{D}: \mathcal{B} X \rightarrow_{\mathcal{B}} X$ is a additive derivation satisfying (25).

For $j=-1$, we can prove the similar stability result. This completes the proof of the theorem.

The following corollary is an immediate consequence of Theorem 4 concerning the stability of (2).

Corollary 5. Let $\lambda$ and $s$ be nonnegative real numbers. If a function $f: \mathcal{B}$ $X \rightarrow_{\mathcal{B}} X$ satisfies the inequality (21) for all $x_{1}, y_{1}, u_{1}, v_{1} \cdots, x_{n}, y_{n}, u_{n}, v_{n} \in_{\mathcal{B}} X$ and

$$
\begin{aligned}
& \| f\left(x_{1} y_{1} \cdots u_{n} v_{n}\right)-f\left(x_{1}\right) y_{1} \cdots \\
& u_{n} v_{n}-x_{1} f\left(y_{1}\right) \cdots u_{n} v_{n}-\cdots-x_{1} y_{1} \cdots u_{n} f\left(v_{n}\right) \| \\
& \leq\left\{\begin{array}{l}
\lambda, \\
\lambda\left\{\left\|x_{i}\right\|^{s}+\left\|y_{i}\right\|^{s}+\left\|u_{i}\right\|^{s}+\left\|v_{i}\right\|^{s}\right\}, \\
\lambda\left\{\prod_{i=1}^{n}\left\|x_{i}\right\|^{s}\left\|y_{i}\right\|^{s}\left\|u_{i}\right\|^{s}\left\|v_{i}\right\|^{s}\right\}, \\
\lambda\left\{\prod_{i=1}^{n}\left\|x_{i}\right\|^{s}\left\|y_{i}\right\|^{s}\left\|u_{i}\right\|^{s}\left\|v_{i}\right\|^{s}\right. \\
\left.+\left(\left\|x_{i}\right\|^{4 n s}+\left\|y_{i}\right\|^{4 n s}+\left\|u_{i}\right\|^{4 n s}+\left\|v_{i}\right\|^{4 n s}\right)\right\},
\end{array}\right.
\end{aligned}
$$

$x_{1}, y_{1}, u_{1}, v_{1} \cdots, x_{n}, y_{n}, u_{n}, v_{n} \in_{\mathcal{B}} X$. Then there exists a unique additive derivation $\mathcal{D}: \mathcal{B} X \rightarrow_{\mathcal{B}} X$ such that

$$
\|f(x)-\mathcal{D}(x)\| \leq\left\{\begin{array}{l}
\frac{\lambda}{|k-1|}, \\
\frac{4 n \lambda|| x||^{s}}{\left|k-k^{s}\right|}, \\
\frac{\lambda|| x||^{4 n s}}{\left|k-k^{4 n s}\right|} \\
\frac{5 n \lambda|| x||^{4 n s}}{\left|k-k^{4 n s}\right|}
\end{array}\right.
$$

for all $x \in \in_{\mathcal{B}} X$, where $k=n^{2}$. 


\section{References}

[1] J. Aczel and J. Dhombres, Functional Equations in Several Variables, Cambridge Univ. Press (1989).

[2] T. Aoki, On the stability of the linear transformation in Banach spaces, $J$. Math. Soc. Japan, 2 (1950), 64-66.

[3] M. Arunkumar and S. Karthikeyan, Solution and stability of $n$-dimensional additive functional equation, International Journal of Applied Mathematics, 25, No 2 (2012), 163-174.

[4] R. Badora, On approximate derivations, Math. Inequal. Appl., 9 (2006), 167-173.

[5] R. Badora, On approximate ring homomorphisms, J. Math. Anal. Appl., 276 (2002), 589-597.

[6] D.G. Bourgin, Approximately isometric and multiplicative transformations on continuous function rings, Duke Math. J., 16 (1949), 385-397.

[7] I.S. Chang, H.M. Kim, On the Hyers-Ulam-Rassias stability of a quadratic functional equations, J. Ineq. Appl. Math., 33 (2002), 1-12.

[8] Chun-Gil Park, On the stability of the linear mapping in Banach modules, J. Math.Anal. Appl., 275 (2002), 711-720.

[9] Chun-Gil Park, modules over a $\mathrm{C}^{*}$-algebra and approximate algebra homomorphisms, J. Math. Anal. Appl., 278 (2003) 93108.

[10] S. Czerwik, Functional Equations and Inequalities in Several Variables, World Scientific, River Edge, NJ (2002).

[11] M. Eshaghi Gordji, H. Khodaei, On the generalized Hyers-Ulam-Rassias stability of quadratic functional equations, Abstr. Appl. Anal. 2009 (2009), Article ID 923476.

[12] M. Eshaghi Gordji, H. Khodaei, R. Khodabakhsh, C. Park, Fixed points and quadratic equations connected with homomorphisms and derivations on non-Archimedean algebras. Adv. in Difference Equations 2012 (2012), Article ID 128.

[13] P. Gavruta, A generalization of the Hyers-Ulam-Rassias stability of approximately additive mappings, J. Math. Anal. Appl., 184 (1994), 431-436. 
[14] D.H. Hyers, On the stability of the linear functional equation, Proc. Nat. Acad. Sci., U.S.A., 27 (1941), 222-224.

[15] K.W. Jun, H.M. Kim, On the stability of an $n$-dimensional quadratic and additive type functional equation, Math. Ineq. Appl., 9, No 1 (2006), 153165.

[16] Pl. Kannappan, Quadratic functional equation inner product spaces, Results Math., 27, No 3-4 (1995), 368-372.

[17] L. Jung-Rye and Sh. Dong-Yun, Isomorphims and derivations in $C^{*}$ algebras, Acta Mathematica Scientia, 31, B(1) (2011), 309-320.

[18] M. Ramdoss, D. Pachaiyappan and H. Dutta, Functional equation and its modular stability with and without $\Delta \mathrm{P}$-condition, Filomat, 34, No 3 (2020), 919-930.

[19] M. Ramdoss, D. Pachaiyappan, I. Hwang and Ch. Park, Stability of an $n$-variable mixed type functional equation in probabilistic modular spaces, AIMS Mathematics, 5, No 6 (2020), 5903-5915.

[20] C. Park, Lie *-homomorphisms between Lie $C^{*}$-algebras and Lie *derivations on Lie $C^{*}$-algebras, J. Math. Anal. Appl., 293 (2004), 419-434.

[21] C. Park, Homomorphisms between Lie $\mathrm{JC}^{*}$-algebras and CauchyRassias stability of Lie $\mathrm{JC}^{*}$-algebra derivations, J. Lie Theory, 15 (2005), 393-414.

[22] C. Park, J. Hou, S. Oh, Homomorphisms between JC*algebras and between Lie $C^{*}$-algebras, Acta Math. Sinica, 21 (2005), 1391-1398.

[23] J.M. Rassias, On approximately of approximately linear mappings by linear mappings, J. Funct. Anal. USA, 46 (1982), 126-130.

[24] Th.M. Rassias, On the stability of the linear mapping in Banach spaces, Proc. Amer. Math. Soc., 72 (1978), 297-300.

[25] P. Narasimman, J.M. Rassias, Ulam-Hyers Stabilities of a generalized composite functional equation in non-Archimedean spaces, Adv. Pure Appl. Math., 7, No 4 (2016), 249-257.

[26] K. Ravi, M. Arunkumar and J.M. Rassias, On the Ulam stability for the orthogonally general Euler-Lagrange type functional equation, International Journal of Mathematical Sciences, 3, No 8 (2008), 36-47. 
[27] S.M. Ulam, Problems in Modern Mathematics, Science Editions, Wiley, New York (1964).

[28] Won-Gil Park, and Jae-Hyeong Bae, Stability of a Bi-Additive Functional Equation in Banach Modules Over a $C^{*}$-Algebra, doi:10.1155/2012/835893.

[29] G. Zamani Eskandani, Hamid Vaezi and Y.N. Dehghan, Stability of a mixed additive and quadratic functional equation in non-Archimedean Banach modules, Taiwanese J. of Math., 14, No 4 (2010), 1309-1324. 